TUNNELLING THROUGH TWO BARRIERS

DMA[IMECC]UNICAMP

\title{
TUNNELLING THROUGH TWO BARRIERS
}

\author{
Stefano De Leo ${ }^{1}$ and Pietro P. Rotelli ${ }^{2}$ \\ 1 Department of Applied Mathematics, State University of Campinas \\ PO Box 6065, SP 13083-970, Campinas, Brazil \\ deleo@ime.unicamp.br \\ 2 Department of Physics, INFN, University of Lecce \\ PO Box 193, 73100, Lecce, Italy \\ rotelli@le.infn.it
}

August, 2004

\begin{abstract}
Recent studies of the tunnelling through two opaque barriers claim that the transit time is independent of the barrier widths and of the separation distance between the barriers. We observe, in contrast, that if multiple reflections are allowed for correctly (infinite peaks) the transit time between the barriers appears exactly as expected.

PACS. 03.65.Xp
\end{abstract}

It is a well known result that the tunnelling time, calculated by using the stationary phase method (SPM) approximation in the limit of an opaque barrier, is independent of the barrier width [1]. Such a phenomenon, called the Hartman effect [2] implies arbitrarily large velocities inside the barrier. When this is obtained with the use of the non-relativistic Schrödinger equation, which can be considered exact only when the velocity of light is infinite, there is no paradox involved, only perhaps some doubt about the relevance. One can in particular question, in this context, the use of the terminology "super-luminal velocities". However, since similar results have more recently been obtained with the Dirac equation $[3,4]$, there is good reason to be perplexed and to invoke further analysis.

We address in this paper a variant of this subject which connects closely to a recent paper upon above-barrier diffusion [5]. In recent years, the Hartman analysis has been extended to a potential model with two successive barriers separated by a free propagation region. Again in the opaque limit for both barriers, it has been observed that, far from resonances, the tunnelling phase time depends neither upon the barrier widths nor upon the distance between the barriers [6,7]. Thus, this result predicts, contrary to common sense, unbounded group velocities even in the free region between the two barriers. This phenomenon, also valid for an arbitrary number of barriers $[8,9]$, is known as the generalized Hartman effect. We shall demonstrate that a different analysis, which allows for multiple scattering between the barriers and, consequently, the existence of multiple peaks, alters this result. Indeed the generalized Hartman effect represents an example of an ambiguity in the use of the stationary phase method.

The starting point of the analysis is the one-dimensional Schrödinger equation

$$
\Psi_{x x}(k, x)=\frac{2 m}{\hbar^{2}}[V(x)-E] \Psi(k, x),
$$

for a particle of mass $m$ in a double barrier potential, 


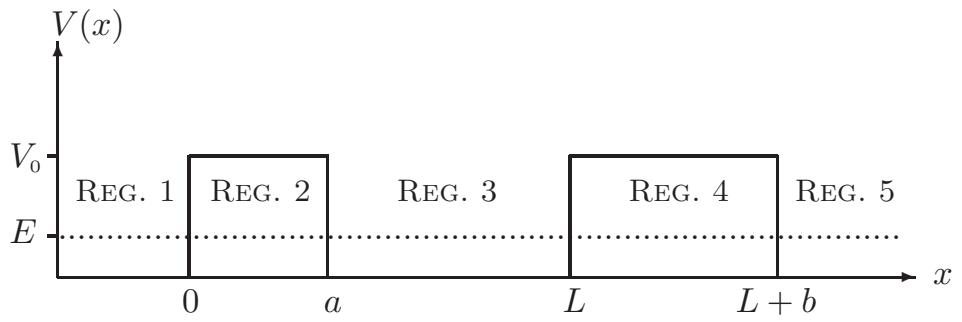

$$
V(x)=\left\{\begin{array}{lr}
0 & x<0 \\
V_{0} & 0<x<a \\
0 & a<x<L \\
V_{0} & L<x<L+b \\
0 & L+b<x
\end{array}\right.
$$

We have maintained different barrier widths because we shall later discuss the case of an opaque limit only for the second barrier but, for simplicity, we have given to the two barriers the same height. It is easy to generalize our formulas to the case of different barrier heights.

The standard procedure for finding the stationary solutions can now be applied. The general solution for $\Psi(k, x)$ and any $E<V_{0}$ in the five regions are

$$
\Psi(k, x)=\left\{\begin{array}{lll}
\text { Region 1: } & e^{i k x}+A_{1 R} e^{-i k x} & {\left[k=\sqrt{2 m E / \hbar^{2}}\right],} \\
\text { Region 2: } & \alpha_{1} e^{-\chi x}+\beta_{1} e^{\chi x} & {\left[\chi=\sqrt{2 m\left(V_{0}-E\right) / \hbar^{2}}\right],} \\
\text { Region 3: } & A_{1 T}\left(e^{i k x}+A_{2 R} e^{-i k x}\right), & \\
\text { Region 4: } & A_{1 T}\left[\alpha_{2} e^{-\chi(x-L)}+\beta_{2} e^{\chi(x-L)}\right], & \\
\text { Region 5: } & A_{1 T} A_{2 T} e^{i k x} .
\end{array}\right.
$$

The requirement of continuity of $\Psi$ and $\Psi_{x}$ at $r=0, a, L$ and $L+b$ give the matching conditions from which, after some algebraic manipulations, we find

$$
\begin{aligned}
A_{1 R} & =\frac{\sinh (\chi a)}{\sinh \left(\chi^{a+2 i \varphi)}\right.}\left[1-\frac{\sinh (\chi b) \sinh (\chi a-2 i \varphi)}{\sinh (\chi a) \sinh \left(\chi^{b+2 i \varphi}\right)} e^{2 i k(L-a)}\right] / \mathcal{D} \\
A_{1 T} & =\frac{2 i \chi k}{w^{2}} \frac{e^{-i k a}}{\sinh \left(\chi^{a+2 i \varphi}\right)} / \mathcal{D} \\
A_{1 T} A_{2 R} & =\frac{2 i \chi k}{w^{2}} \frac{\sinh (\chi b) e^{i k(2 L-a)}}{\sinh \left(\chi^{a+2 i \varphi) \sinh (\chi b+2 i \varphi)} / \mathcal{D}\right.} \\
A_{1 T} A_{2 T} & =\left(\frac{2 i \chi k}{w^{2}}\right)^{2} \frac{e^{-i k(a+b)}}{\sinh \left(\chi^{a+2 i \varphi}\right) \sinh \left(\chi^{b+2 i \varphi}\right)} / \mathcal{D}
\end{aligned}
$$

where

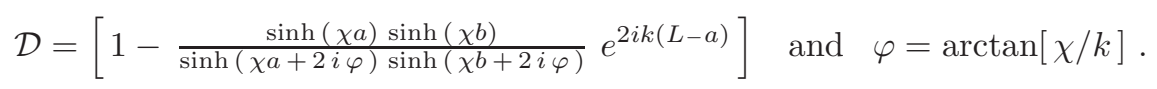

If we consider the (double) opaque limit, $\chi a$ and $\chi b \gg 1$, we reproduce (when $a=b$ ) the coefficients which have been used to obtain the generalized Hartman effect $[6,7,9]$,

$$
\begin{aligned}
A_{1 R} & \approx \exp [-2 i \varphi], \\
A_{1 T} & \approx \frac{2 \chi k}{w^{2}} \exp [-\chi a-i k L] / \sin [2 \varphi-k(L-a)], \\
A_{1 T} A_{2 R} & \approx \frac{2 \chi k}{w^{2}} \exp [-\chi a+i k L-2 i \varphi] / \sin [2 \varphi-k(L-a)], \\
A_{1 T} A_{2 T} & \approx \frac{8 i \chi^{2} k^{2}}{w^{4}} \exp [-\chi(a+b)-i k(L+b)-2 i \varphi] / \sin [2 \varphi-k(L-a)] .
\end{aligned}
$$

We recall that all the above amplitudes are to be multiplied by the plane-wave phase factor and the chosen modulation function assumed peaked at $k=k_{0}$. Without repeating all the arguments leading to the generalized Hartman effect (including the SPM itself), it suffices to note that, in the expression for $A_{1 T} A_{2 T}$ of Eqs.(44), the $L+b$ dependence in the phase will be cancelled by the plane wave phase calculated at $x=L+b$. There remains an $L-a$ dependence in the modulus but that does not affect the time for the appearance of the maximum in region 5, under the hypothesis of a single peak .

Previous analysis upon the generalized Hartman effect can be criticized on several grounds. One objection is the standard use in the through-going phase of the peak momentum $k_{0}$ of the incoming wave. The Hartman time for transit through a single barrier then reads as $\tau_{0}=2 \varphi^{\prime}\left(k_{0}\right)$, where the prime stands for differentiation with respect to $k$. This may be a good approximation for narrow barriers, but it is generally wrong for opaque barriers because the peak or maximum value in the outgoing momentum amplitude is not the same as that of the modulation function (incoming amplitude). The only exception is if the modulation function, which transforms the sum of plane waves into wave packets, is so sharply truncated that only momentum close to the incoming maximum are 
involved. What we like to call the "filter effect" of a barrier preferentially allows the higher momentum components to transit. So, in the opaque limit, only the highest incoming momentum passes through. For example, the group velocity in region $5, \tilde{k}_{0} / m$, is always higher than the incoming group velocity $k_{0} / m$. A more precise expression for the Hartman time is thus $\tilde{\tau}_{0}=2 \varphi^{\prime}\left(\tilde{k}_{0}\right)$.

Another principal criticism is the implicit assumption of a single reflected and transmitted wave packet. Let us list some objections:

1) There is never any theoretical reason given for this assumption.

2) A natural alternative exists which still uses the SPM but involves multiple reflections with the "expected" transit times (see below).

3) The momentum distribution of the transmitted wave clearly displays multiple momentum peaks.

4) For technical reasons it is non trivial to resolve numerically the twin barrier problems in the opaque limit in which formally the transmitted probability goes to zero. However, if, for example, only the second barrier is made opaque and the first is kept narrow, it is very easy to perform numerical calculations and see the multiple peaks in the reflected amplitude. Similarly for the foreward/backward flowing peaks in the intermediate region 3 [10].

Now for the alternative approach. Before going to the opaque limit, the denominator factor $\mathcal{D}$ in Eqs.(3i), can be legitimately expanded as a series in the numerator. In particular, in region 5, we obtain the following transmitted amplitude

$$
\begin{aligned}
A_{1 T} A_{2 T}= & \left(\frac{2 i \chi k}{w^{2}}\right)^{2} \frac{e^{-i k(a+b)}}{\sinh \left(\chi^{a}+2 i \varphi\right) \sinh \left(\chi^{b+2 i \varphi}\right)} \times \\
& \sum_{n=1}^{\infty}\left(\frac{\sinh (\chi a) \sinh (\chi b)}{\sinh (\chi a+2 i \varphi) \sinh \left(\chi^{b}+2 i \varphi\right)} e^{2 i k(L-a)}\right)^{n-1} .
\end{aligned}
$$

An (infinite) sum of terms each of which can be analyzed with the SPM to determine the position of their maxima. This is just the procedure used in ref. [5]. The phase of the first transmitted peak $(n=1)$ is given by

$$
\Phi_{1}=k x-E t-k(a+b)-\arctan [\tan (2 \varphi) \operatorname{coth}(\chi a)]-\arctan [\tan (2 \varphi) \operatorname{coth}(\chi b)] .
$$

Consequently, the first transmitted peak appears at $x=L+b$ in perfect accord with the expected $(L-a$ dependent $)$ transit time. The dependence of the subsequent phases $(n=2,3 \ldots)$,

$$
\Phi_{n}=\Phi_{1}+(n-1)\{2(L-a)-\arctan [\tan (2 \varphi) \operatorname{coth}(\chi a)]-\arctan [\tan (2 \varphi) \operatorname{coth}(\chi b)]\},
$$

contain extra multiples of $2(L-a)$. In fact, in the opaque limit

$$
\Phi_{n} \approx k x-E t-k(a+b)-4 \varphi+(n-1)[2(L-a)-4 \varphi],
$$

the filter effect of the first barrier guarantees that the group velocity in region 3 is the same as that in region 5. Thus, the successive exit times for the maxima predicted by the SPM are proportional to multiples of the back/foreward travel times in the inter-barrier region plus multiples of twice the Hartman time,

$$
\left.t_{n}=\left(m / \tilde{k}_{0}\right)\left[(2 n-1)(L-a)+2 n \tilde{\tau}_{0}\right)\right] .
$$

The multiples of the Hartman time that appear above are a consequence of the multiple doublereflections. The so-called "delay-time" in reflection in region 3 coincides with the Hartman transit time in the opaque limit since the relevant momentum used in the SPM for both is the upper limit $\tilde{k}_{0}$.

We have tested, but will not demonstrate here, that even though the outgoing amplitudes are essentially independent of the shape of the assumed modulation function (since they are dominated by the exponential factors of the hyperbolic-sine functions) the SPM still works surprisingly well. The only improvement we would suggest, given the asymmetry in the momentum distribution, is the use of the average momentum value in place of the peak momentum value.

There is an objection that can be made to our analysis so far. We have not demonstrated that the transmitted wave packets are not overlapping and hence the treatment of each separately may be wrong. Indeed, we shall now argue that in the opaque limit the outgoing wave packets do overlap substantially. Nevertheless, we still claim that our treatment is more sensible than that of a singlewave SPM analysis. To determine the size of the outgoing wave packets, we employ the Heisenberg 
uncertainty principle. In the opaque limit, the dominating part of the modulus of each of the outgoing wave-packets is given by the filter term: $\exp [-\chi(a+b)]$. This means that $\Delta \chi=1 /(a+b)$. Consequently, $\Delta k=\langle\chi / k\rangle \Delta \chi \ll 1 /(a+b)$. It follows that $\Delta x \gg(a+b)$. Thus, in the opaque limit, for any fixed value of the inter-barrier separation $L-a$, the wave packet widths will eventually exceed the separation between successive peaks. Now the successive wave peaks of the outgoing amplitude are, in the opaque limit, almost equal in magnitude. For example in the case when interference does not shift these maxima (low interference) a resulting "dragon-like" structure emerges. The highest first maximum appears, as given in Eq.(6), at the transit time in region 3 plus the two Hartman times (because of two barriers). However, one must ask what physical significance can be given to this maximum. The only definition of position that is reasonable (for broad wave packets) is the mean position of the wave packet and this appears at a very different time from the maximum (first peak). It is the asymmetry predicted by our interpretation in the transmitted wave train that makes this question of mean vs. maximum relevant, and distinguishes our procedure from a single wave analysis.

In conclusion, we have shown that the generalized Hartman effect is the consequence of treating the outgoing wave as a single wave packet. For us, this is not the natural choice as we have explained

above. Even when the multiple outgoing wave packets overlap to the extent that they appear as a single wave packet, the position of the maximum is far removed from the mean position and is hence of little physical significance.

\section{References}

1. V. S. Olkhovsky V. S. and E. Recami, Phys. Rep. 214, 340 (1992).

2. T. E. Hartman, J. Appl. Phys. 333427 (1962).

3. P. Krekora, Q. Su and R. Grobe, Phys. Rev. A 63, 032107 (2001); ibidem 64, 022105 (2001).

4. V. Petrillo and L. Refaldi, Phys. Rev. A 67, 012110 (2003).

5. A. E. de Bernardini, S. De Leo and P. Rotelli, "Above barrier potential diffusion" (quant-ph/0408028).

6. V. S. Olkhovsky, E. Recami and G. Salesi, Europhys. Lett. 56, 879 (2002).

7. E. Recami, J. Mod. Opt. 15, 913 (2004).

8. Y. Aharonov, N. Erez and B. Reznik, Phys. Rev. A 65, 052124 (2002).

9. S. Esposito, Phys.Rev. E 67, 016609 (2003).

10. Examples and plots relative to these last two points for specific parameters and modulation functions will be presented elsewhere. 